\title{
Applications of the 1000-turns Orbit Measurement System at LEP.
}

\author{
P. Castro*, DESY-Hamburg, Germany
}

\begin{abstract}
The software of the LEP beam orbit measurement system [1] allows the acquisition of the beam position for over 1000 consecutive turns. The acquisition can be synchronized to a deflection by a kicker in order to observe coherent betatron oscillations. In this paper we describe the use of this technique to study the dependence of head-tail damping on beam parameters and the tune dependence on the oscillation amplitude for various LEP optics [2]. Coherent oscillations can be represented in phase space to observe the effect of resonances and other non-linear behavior $[4,5]$. Coherent oscillations can also be excited using a sinusoidal deflection in order to maintain the oscillation amplitude [6]. Thus, precise measurements of the phase advance are obtained by harmonic analysis of the signal at each pick-up. This technique has been used to measure the beam energy dependence of the phase advance due to chromaticity [7] and the bunch current dependence of the phase advance due to impedance [8]. Finally, we describe a method to obtain the betatron function from the measured phase advance and report about its accuracy and applications at $\operatorname{LEP}[9,10]$.
\end{abstract}

\section{INTRODUCTION}

Measurements of the beam position turn-by-turn and their storage over many turns have many useful applications and have been relevant to LEP performance improvements [11]. The possibility of triggering these measurements on preprogrammed timing signals provide a valuable tool to study the trajectory of the beam at injection and during the energy ramp. This application is particularly helpful in order to establish a first circulating beam after a long shutdown. In superconducting proton machines like HERA, this feature is also used to store the beam orbit at the last turns before the beam is dumped in case of anomalous beam particle losses (in order to prevent further loss on superconducting magnets). In that way it is possible to diagnostic the cause of such losses.

In this report we present some of the recent applications of 1000-turns measurements involving the observation of beam coherent betatron oscillations at LEP. These measurements provide qualitative and quantitative measurements of linear and non-linear beam optics properties.

At LEP there are 504 pick-ups located around the circumference. On each turn and for every bunch the signals of the pick-ups are processed and stored in a memory buffer with a capacity to store up to 1024 turns. Beam coherent oscillations are excited using a fast bending mag-

\footnotetext{
*Email: pcastro@mail.desy.de
}

net or kicker. A single transverse deflection can be applied to the beam in order to observe the damping of coherent oscillations. Studies of the amplitude decay of coherent oscillations were carried out to determine the contribution of head-tail damping and radiation damping and the horizontal detuning with amplitude at LEP [2]. The kicker can also introduce beam deflections on each turn with an amplitude that follows a sinusoidal curve at any given frequency. If the frequency of the continuous excitation is close to the betatron tune the beam will oscillate coherently and within a very short time an almost constant amplitude can be reached. A measurement of betatron phase advance between pick-ups can be obtained by harmonic analysis of these data. The measured phase advance between three pick-ups can be used to evaluate the optics parameters $\beta$ and $\alpha$ at any azimuthal position around the storage ring [10].

\section{MEASUREMENTS OF COHERENT DAMPING}

The damping of coherent beam oscillations and the shift of its frequency can be observed using 1000-turns measurements. The measurement technique consists in triggering the 1000-turn acquisition a few turns before a single kick to the beam is applied. This permits to observe the start and damping of the coherent beam oscillations. An example of such measurement is shown in fig. 1.

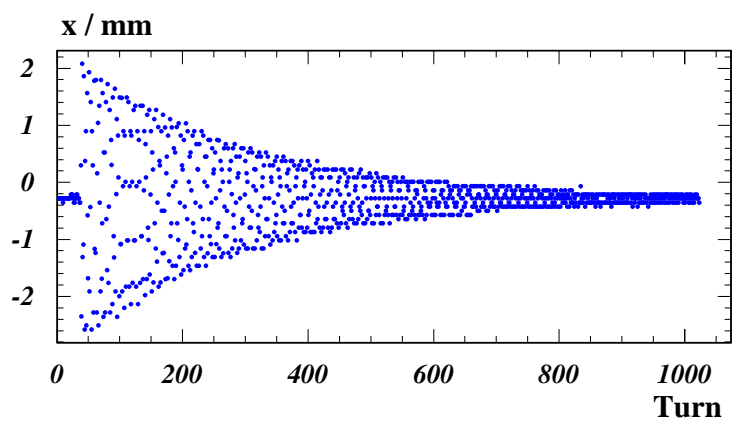

Figure 1: Measured center-of-charge position of a bunch versus turn number. A single kick was applied a few turns after the acquisition started.

A fit to the data using a damped oscillation with amplitude dependent frequency yields the coherent damping time $\tau$. At LEP the coherent damping is a contribution of radiation and head-tail damping

$$
\frac{1}{\tau}=\frac{1}{\tau_{o}}+\frac{1}{\tau_{\text {head-tail }}}
$$


At a given energy the head-tail damping is proportional to the chromaticity $Q^{\prime}$ and to the bunch current $I_{\text {bunch. In }}$ general the head-tail damping is dominating and its contribution can be determined by measuring the coherent damping rate at bunches with different currents. The radiation damping rate can be extracted by an extrapolation to zero bunch current. A dedicated experiment was carried out at LEP [2] where the coherent damping rate was measured for various bunch currents and chromaticities at beam energies of 45.625 and $60.0 \mathrm{GeV}$. As an example, measured coherent damping rates as function of bunch current are plotted in fig. 2. With the radiation damping time, the horizontal damping partition number $J_{x}$ is determined. The measured $J_{x}$ agrees with results of direct measurements of the circumference using pick-ups and tide models [12]. This measurement plays an important role in the understanding of the LEP beam energy since the $J_{x}$ shifts are accompanied by an energy shift.

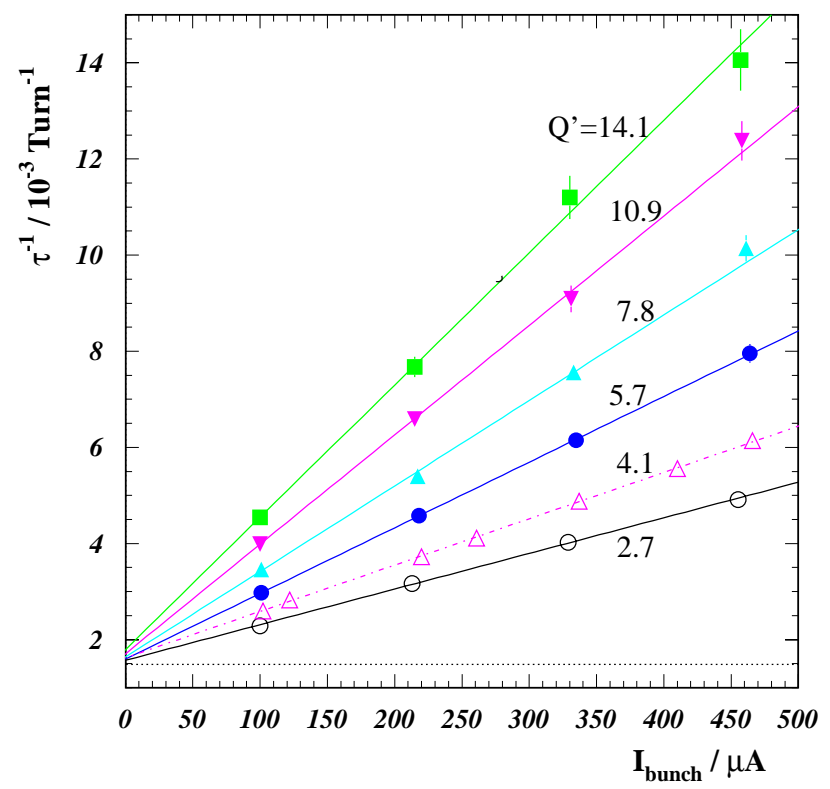

Figure 2: Coherent damping rate versus bunch current measured at a beam energy of $45.625 \mathrm{GeV}$ for several chromaticities.

\section{TUNE SHIFT WITH AMPLITUDE}

At LEP the fractional part of the horizontal betatron tune $Q_{x}$ is about 0.28 and a large tune spread in the beam can drive some of the particles onto the third order resonance. A large detuning with amplitude (anharmonicity) limits therefore the amplitude range of stable particles, i.e. the aperture for the beam.

The detuning with amplitude can be extracted from 1000-turns measurements of damped betatron oscillations. The amplitude decay $A=A_{0} \mathrm{e}^{-t / \tau}$ is obtained from a "global" fit to the damped oscillation. Taking subsamples of several turns we obtain the tune evolution as a function of the turn number. An example of the results of such fit is shown in fig. 3. At the second half (above 500 turns) the oscillation amplitude is small and the results of the fit is less precise.

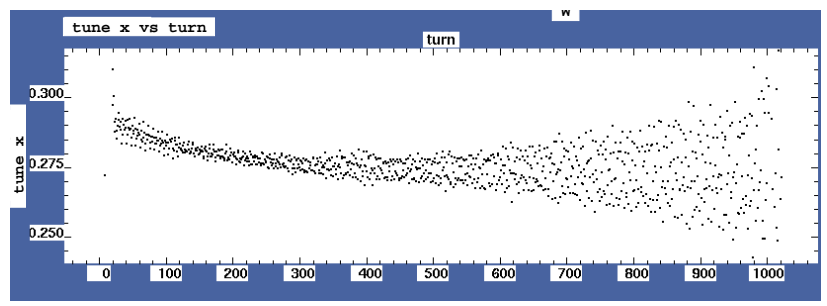

Figure 3: Detuning results as a function of turn number.

The horizontal detuning is a linear function of the Courant-Snyder invariant $W$ defined as:

$$
W=\frac{1+\alpha^{2}}{\beta} x^{2}+2 \alpha x x^{\prime}+\beta x^{\prime 2} \simeq \frac{A^{2}}{\beta}
$$

where $\beta$ is the value of the betatron function at the pickup and $\alpha=-(1 / 2)(d \beta / d s)$. This linearity can be seen in fig. 4.

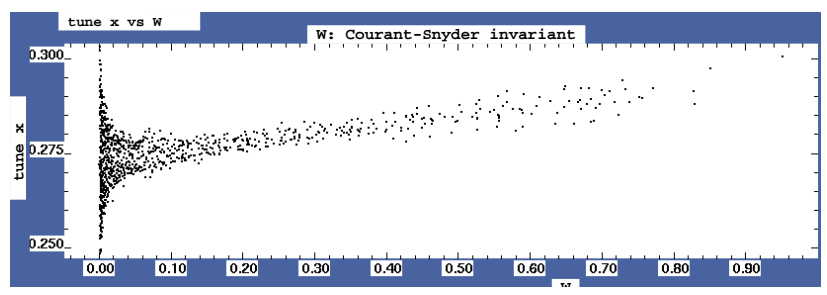

Figure 4: Detuning results as a function of the CourantSnyder invariant $W$.

The detuning with amplitude $\left(\partial Q_{x} / \partial W\right)$ is obtained from a fit to the tune versus $W$. This procedure is applied to a large number of pick-ups to obtain a better statistics. Results of such measurements for three LEP physics optics are given in [2,3] and confirms predictions of the LEP model.

\section{PHASE SPACE REPRESENTATION}

Coherent betatron oscillations in presence of resonances are studied in phase space diagrams. In these diagrams the beam is represented on each turn by a point with coordinates $\left(x, x^{\prime}\right)$, where $x$ is the displacement and $x^{\prime}=d x / d s$ is approx. the angle of the beam trajectory with respect to the design orbit ( $s$ is the longitudinal coordinate). The traditional way for representing the 1000-turns measurements in phase space consists in finding two pick-ups separated by a phase advance of $90^{\circ}$. When the phase advance is exactly $90^{\circ}$ the signal at the second pick-ups is proportional to $(d x / d s)$ at the first pick-up. However, beta-beating can introduce errors in the interpretation of the data.

The approach followed in $[4,5]$ consists in using the 1000-turns measurement of a single pick-up and rotating by $90^{\circ}$ its Fourier transform to create a "virtual" pick-up. A betatron oscillation (even when it is damped) can be well described in terms of harmonic functions. Therefore, a 
phase shift of $90^{\circ}$ is equivalent to a differentiation operator. The procedure starts by performing a Fourier transformation to the 1000-turns data and obtaining an array of 1000 complex values (sine and cosine components of each frequency). Rotation by $90^{\circ}$ means simply an exchange of values between real and imaginary parts and change of sign in the real (cosine) part. In order to reduce errors due to the discreteness (sampling) and finiteness (window) of data, a filter is introduced. The filter used is basically a linearly decreasing weight at frequencies below 0.12 and above 0.39 (in tune units). The 1000-turns of the virtual pick-up is obtained by a reverse Fourier transformation on the rotated and filtered complex array. A phase space representation is then obtained by plotting the positions of the virtual pickup against the ones of the real pick-up, centering around the origin and normalizing by $1 / \sqrt{\beta}$. An example of such a plot when kicking the beam with a tune slightly above $2 / 7$ is shown in fig. 5. One observes in this figure the typical arms that are formed when the betatron tune is close to a resonance.

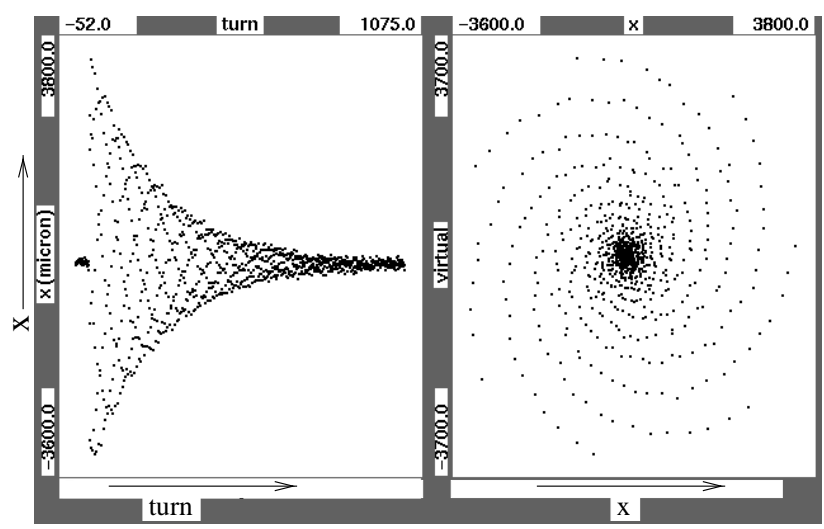

Figure 5: Coherent oscillation amplitude versus turn (left) and in phase space (right). The tune is slightly above 2/7. (Phase space not normalized by $1 / \sqrt{\beta}$.)

The behavior of the coherent beam oscillations at LEP have been observed under several conditions (tunes, coupling) and at different beam energies. Effects like beam trapped in a 3rd order resonance and partially lost, crossing higher order resonances, coupling between horizontal and vertical motion, filamentation and other non-linearities have been reported in $[4,5]$.

\section{BETATRON PHASE MEASUREMENTS}

As already seen in section 2 the amplitude of coherent oscillations decreases rapidly within a few hundred turns mainly due to head-tail damping. In order to compensate this damping effect and maintain a coherent betatron oscillation with more or less constant amplitude we need to keep the transverse excitation. The kicker used at LEP can provide this excitation with a sinusoidal modulation of its field. If the frequency is close to the fractional part of the tune, coherent oscillations are excited and after a short time reach a constant amplitude. This amplitude depends on the strength of the sinusoidal deflection and the proximity of the frequency to the tune. For a precise measurement of the phase of these oscillations, the beam must be excited to high amplitude to gain in signal to noise ratio. However, the presence of non-linear elements (like sextupoles, etc.) limits the valid amplitude range of this measurement.

The 1000-turns obtained during coherent oscillations of constant amplitude are then harmonically analyzed to give the amplitude and phase of the oscillation at each pick-up. The systematic error on the phase measurement is a function of the longitudinal size of the beam. At LEP, this systematic error is negligible because the bunch length is about $1 \mathrm{~cm}$. The statistical error of the phase $\sigma_{\phi}$ is given by [9]

$$
\sigma_{\phi}=\frac{1}{A} \sqrt{\frac{2}{N}} \sigma_{x}
$$

where $A$ is the amplitude of the oscillation, $N$ the number of samples (or turns, in our case 1024) and $\sigma_{x}$ is the statistical error of position measurements of the pick-up. For a typical oscillation amplitudes at LEP of around $2 \mathrm{~mm}$ and position resolution (turn-by-turn) of $\sigma_{x} \simeq 0.1 \mathrm{~mm}$ the error of phase measurements is about $0.13^{\circ}$, i.e. the error on phase advance measurements is about $0.2^{\circ}$.

\subsection{Applications of betatron phase measure- ments}

Betatron phase measurements have been applied to check the chromaticity correction of the magnetic lattice of LEP [7] and the distribution of impedance of the vacuum chamber around the circumference of LEP [8]. The first was carried out by shifting the RF-frequency (which changes the length of the closed orbit and consequently the beam energy) and taking phase measurements at various beam energies. The results clearly show the negative chromaticity produced in the straight sections and its correction in the arcs. The agreement between experiment and calculation is very good and exclude strong localized sextupole errors.

The measurement of the impedance distribution was achieved by comparing phase measurements with bunch currents ranging from 50 to $450 \mu \mathrm{A}$. The results are in good agreement with the impedance model of LEP. These measurements clearly show the localized impedance contribution of copper cavities by a sudden phase shift around straight sections 2 and 6 . In the rest of the machine the phase shift presents a rather constant slope due to the influence of bellows. As K. Cornelis put it once in the 95' CAS school: "It is indeed a very sophisticated way to find out the location of the accelerating cavities along the $26 \mathrm{~km}$ of LEP circumference".

\section{BETATRON FUNCTION MEASUREMENTS}

The measured phase advances between the pick-ups can be used to evaluate the optics parameters at any azimuthal position around the circumference $[10,9]$. Taking a given 
pick-up and the ones adjacent and using the three measured phase advances along with the theoretical phase advances coming from a tracking program such as MAD [13] one obtains the ratio of the real $\beta$ value to the theoretical one:

$$
\frac{\beta_{2}}{\beta_{2}^{t}}=\frac{\cot \phi_{12}+\cot \phi_{23}}{\cot \phi_{12}^{t}+\cot \phi_{23}^{t}}
$$

where ${ }^{t}$ denotes theoretical values, $\beta_{2}$ is the value of the betatron function at the 2 nd pick-up, $\phi_{12}$ is the phase advance between 1 st and 2nd pick-up and $\phi_{23}$ between 2 nd and 3rd pick-up. Equivalent expressions are obtained for the 1st and 3rd pick-up. These expressions can also be written replacing the theoretical values by the second elements of the transfer matrices between the pick-ups. Thus,

$\beta_{2}=\frac{a_{12} b_{12}}{c_{12}}\left(\cot \phi_{12}+\cot \phi_{23}\right)=\frac{a_{12} b_{12}}{c_{12}} \frac{\sin \phi_{13}}{\sin \phi_{23} \sin \phi_{12}}$

where $a_{12}, b_{12}$ and $c_{12}$ are the second element of $A$ (transfer matrix between 1st and 2nd pick-up), of $B$ (between 2nd and 3rd pick-up) and of $C=B \times A$, respectively.

When the transfer matrices between the three pick-ups are known, we obtain the relative phase advance $\phi(s)=$ $\mu(s)-\mu\left(s_{1}\right)$ at any $s$ between the three pick-ups:

$\phi(s)=\arctan \left[\frac{a_{12} n_{12}(s)}{b_{12} m_{12}(s)} \cot \phi_{12}-\frac{c_{12} o_{12}(s)}{b_{12} m_{12}(s)} \cot \phi_{13}\right]$

where $m_{12}(s), n_{12}(s)$ and $o_{12}(s)$ are the second elements of the matrices $M(s), N(s)$ and $O(s)$ defined in fig. 6 .

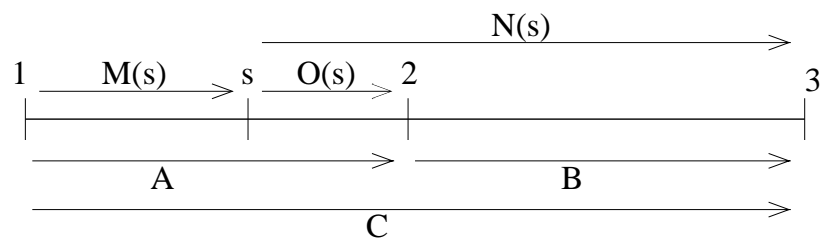

Figure 6: Matrix notation used in the equations.

The value of $\beta$ at $s$ is given by

$$
\beta(s)=\frac{b_{12} m_{12}^{2}(s)}{a_{12} c_{12}} \frac{\sin \phi_{12} \sin \phi_{13}}{\sin \phi_{23} \sin ^{2} \phi(s)}
$$

If the theoretical values are known, then the ratio of the real $\beta$ value to the theoretical one at $s$ is

$$
\frac{\beta(s)}{\beta^{t}(s)}=\frac{\beta_{1}}{\beta_{1}^{t}} \frac{\sin ^{2} \phi(s)}{\sin ^{2} \phi^{t}(s)}
$$

An example of such measurements is shown in fig. 7 . The values of the betatron function are obtained by "advancing" around the circumference from one central pickup to the next.

\subsection{Error analysis}

If the transfer matrices are correct, i.e. the perturbation which is causing the beta-beating is outside the three chosen pick-ups, this procedure yields correct values only affected by the statistical error of the phase measurements.

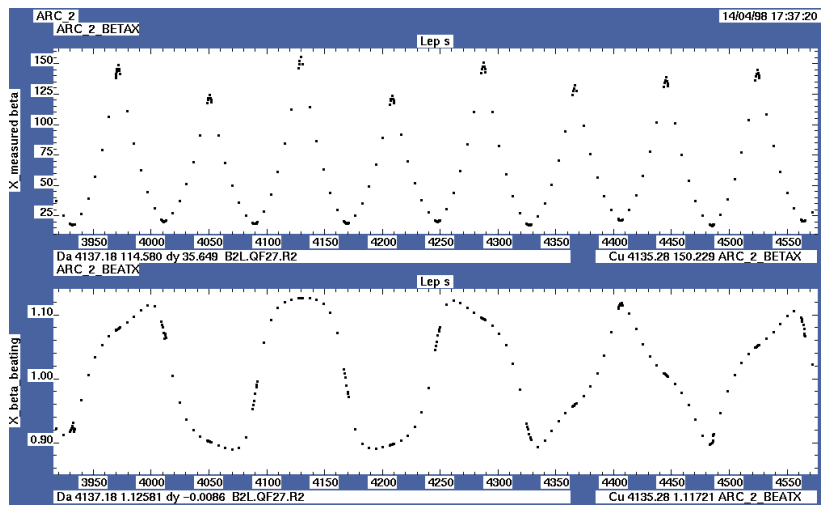

Figure 7: Example of measured horizontal $\beta$ (top) and betabeating $\beta / \beta^{t}$ (bottom) in the arcs

At LEP the perturbations arise in the insertion regions, so this procedure provides precise values of $\beta$ in the arcs. In the arcs the systematic error in the measurement of $\beta$ from relative errors of quadrupole gradients of about $5 \cdot 10^{-4}$ has been estimated to be about $0.2 \%$.

The contribution of the statistical error of the phase measurement on the measured $\beta$ depends on the phase advances between the three pick-ups. If the phase advance is around $60^{\circ}$, we have

$$
\varepsilon_{\beta_{2}}=\sqrt{\frac{8}{3}} \sigma_{\phi} \simeq 1.63 \sigma_{\phi}
$$

assuming that the measurement errors of the phase are equal at the three pick-ups. For a $\sigma_{\phi}=0.13^{\circ}$, the relative error on the measured $\beta$ is about $0.4 \%$. The statistical error of $\beta$ measurements is in the order of $0.5 \%$ in a range of phase advances from $\sim 30^{\circ}$ to $\sim 75^{\circ}$. Outside this range, the error increases exponentially until the phase advance is 0 or $90^{\circ}$.

\subsection{Applications of betatron function measure- ments}

Betatron functions measurements show the mismatch of the betatron function with respect to the theoretical values (also called beta-beating or beta-wave). In terms of $\left(\beta / \beta^{t}\right)$ this mismatch appears as an oscillation with a phase twice of the phase advance. An example of a vertical beta-beating measurement is shown in fig. 8 . The beta-beating observed is of the order of $10 \%$ and up to $20 \%$ in some places. The curve is not drawn at places where the pick-up readings were not available during this measurement. In this figure we observe rapid changes of the beta-beating at the Interaction Points (IP) 2 and 4. The changes in the amplitude of the beta-beating observed at IP 2 and 4 indicate perturbations of the betatron function. As expected, beta-beating is introduced at the four low- $\beta$ insertions (IP 2, 4, 6 and 8).

Phase advance measurements show also a similar mismatch as the betatron function. In fact, a "phase-beating" is also observed in the difference between measured and theoretical phase advance. The measured phase-beating plotted as a function of the theoretical phase advance in fig. 9 


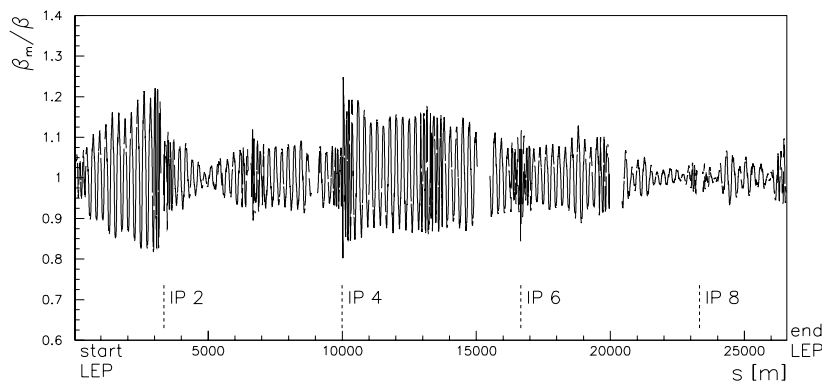

Figure 8: Measured vertical beta-beating.

corresponds to the same measurement of the beta-beating shown above in fig. 8. Phase advance changes at IP 2 and 4 indicate a local strong quadrupole gradient error.

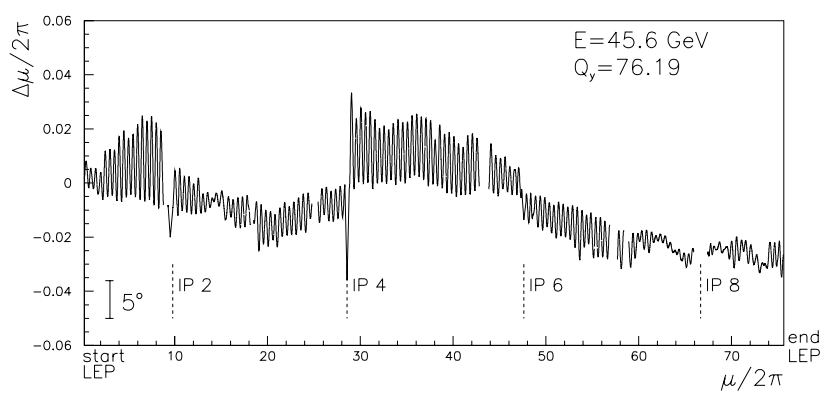

Figure 9: Measured vertical phase-beating.

Betatron function measurements have been extensively applied to calculate emittances from transverse beam profile measurements at LEP. Beta-beating introduces a systematic error on emittance measurements if only the theoretical betatron function is taken. Wire scanners at LEP, which are equipped with a high precision ruler, provide an accurate absolute measurement of the size of the beam profile. The measured values of the betatron function were applied in order to use the wire scanner emittance measurement as a reference to calibrate synchrotron light imaging monitors [15].

The strongest contribution to the beta-beating are quadrupole gradient errors. A smaller contribution has its origin in the beam energy changes around the circumference due to synchrotron radiation losses at the bending magnets. This energy loss is recovered in the RF cavities installed symmetrically around IPs 2 and 6 . At $45.6 \mathrm{GeV}$ the effect on the beta-beating is up to $6 \%$ and it is opposite for each type of particles. Measurements of the betatron function carried out with the electron and the positron beam were compared and its difference resulted in a $\left[\beta\left(e^{-}\right)-\beta\left(e^{+}\right)\right] / \beta$ with an amplitude of up to $12 \%$. This is in very good agreement with the predictions [10].

In 1993 and 1994 the number of bunches per beam was increased to eight with the "pretzel" scheme [14]. In order to avoid beam encounters in the middle of the arcs, beams were separated in the horizontal plane and displaced up to about $4 \mathrm{~mm}$ from the design orbit. This separation introduces beta-beating, because of the large excursions of the beam off-center of the sextupole magnets which act on the beam as an extra quadrupole gradient. Since the offset of the positron and the electron beam are just opposite, the beta-beating has also an opposite sign. Measurements of the betatron function were done with a single beam with and without the pretzel separation and confirmed the predictions for beta-beating of $20 \%$ and up to $30 \%$ due to the separation.

\section{ACKNOWLEDGMENTS}

Many thanks to all who have contributed to the results presented above and for all the helpful and instructive discussions. Especially I would like to thank A.-S. Müller and G. Morpurgo for their help in preparing the presentation as well as this paper.

\section{REFERENCES}

[1] G. Morpurgo, "The Software for the CERN LEP Orbit Measurement System", CERN-SL-91-41, (1991).

[2] A.-S. Müller and J. Wenninger, "Measurements of Coherent Damping and Tune Shifts with Amplitude at LEP", Proc. of the 6th EPAC, Stockholm, (1998).

[3] A.-S. Müller and J. Wenninger, "Measurements of Tune Shifts with Amplitude at LEP", poster THA129 at this conference.

[4] G. Morpurgo, "The BOM Turn Display: A Tool to Visualize the Transverse Phase-Space Topology at LEP", Proc. of the 6th EPAC, Stockholm, (1998), pp.1571-3, and CERN SL-98045 (BI).

[5] G. Morpurgo, "Measurement and Visualization of the Transverse Phase-Space Topology at LEP", Proc. of the ICAP, Monterey, CA, (1998).

[6] J. Borer et al., "Harmonic Analysis of Coherent Bunch Oscillations in LEP", Proceedings of the 3rd EPAC, Berlin, (1992), pp. 1082-4.

[7] D. Brandt et al., "Measurement of Chromatic Effects in LEP", Proceedings of the 1995 PAC, Dallas, USA, 1995.

[8] D. Brandt et al., Measurement of Impedance Distributions and Instability Thresholds in LEP; Proc. of the 1995 PAC, Dallas, USA, 1995.

[9] J. Borer et al., "Betatron Function Measurement at LEP Using the BOM 1000 Turns Facility", Proc. of the 1993 PAC, Washington D.C., (1993), pp. 2103-5.

[10] P. Castro, "Luminosity and beta function measurement at the electron-positron collider ring LEP", Doctoral Thesis, CERN SL/96-70 (BI) (1996).

[11] S. Myers, "Performance Related Measurements on LEP", CERN-SL-99-002 DI, (1999).

[12] P. Melchior, "Tidal Interactions in the Earth Moon System", Communications, Serie B, No. 160, IUG General Assembly, Vienna, (1991).

[13] H. Grote and F.C. Iselin; "The MAD program", CERN/SL/90-13(AP), (1991).

[14] L. Bergström et al., "Report of the Working Group on High Luminosities at LEP", Editors E. Blucher, J. Jowett, F. Merrit, G. Mikenberg, J. Panman, F.M. Renard and D. Treille. CERN 91-02, (1991).

[15] R. Jung, "Precision emittance measurements in LEP with imaging telescopes, comparison with wire scanner and $\mathrm{x}$ ray detector measurements", Proc. of the Workshop on Particle Dynamics in Accelerators, Tsukuba (Japan), (1994), and CERN-SL-95-63 (BI). 